\title{
A Hybrid Model for Human Recognition System Using Hand Dorsum Geometry and Finger-Knuckle-Print
}

\author{
${ }^{1}$ B. Mathivanan, ${ }^{2}$ V. Palanisamy and ${ }^{3}$ S. Selvarajan \\ ${ }^{1}$ Department of CSE, Sri Ramakrishna Engineering College, Coimbatore, India \\ ${ }^{2}$ Information Institute of Engineering, Coimbatore, India \\ ${ }^{3}$ Muthayammal Technical Campus, Rasipuram, India
}

Received 2011-09-23, Revised 2012-09-06; Accepted 2012-09-08

\begin{abstract}
This study focuses on developing an efficient person identification and recognition system using hand based biometrics for secured access control. In most of the previous works on hand-based recognition methods, mostly, the importance was not given to the top side of the hand, which is used in this model. Iin our previous work we have developed a Hand based biometric recognition system using the palm side of the hand. In which, all features were extracted only from the palm side of the hand. Also, in some of the earlier works, the palm side of the hand was used for recognition purpose. The reason behind the selection of palm side of the hand is, it is very easy to capture using a simple scanning device and we can extract the shape based features as well as the palm print from the same image. In this study, we address a new hybrid model for biometrics based human recognition system using the dorsum of hand and the finger knuckle print. Dorsum of hand (backside of hand or topside of hand) is the opposite side of the palm side of the hand. In this study, we highlight some of the advantages of using dorsum of hand for modeling a biometrics based human recognition system. This study proposes a new hybrid model biometric system using Dorsum of Hand. Both the finger knuckle print and hand shape features are proposed to be extracted from the single hand image acquired from a top mounted camera setup. We use some unique features that improve the accuracy of the recognition. Several more significant hand attributes that can be used to represent hand shape and improve the performance are examined. Effective algorithms were used in the process of extracting different kinds of salient features from the dorsum of hand image.
\end{abstract}

Keywords: Biometrics Based, Hand Geometry, Short Periods, Human Recognition, Finger-Knuckle-Print, Personal Authentication, Current Research, Hybrid Model, Human Hand

\section{INTRODUCTION}

Reliability in personal authentication system is key to the security in the networked community. Many bodily characteristics of human, i.e., biometrics, are typically time invariant, unique and easy to acquire for every individual. In many of the access control systems, Biometric features such as face, palm print, signature, iris, fingerprint, hand geometry have been suggested for the security. Many of the biometric based current researches have been focused on fingerprint and face. The accuracy and reliability using face is currently low as the researchers today continue to tackle with the problems of pose variation, lighting, gesture and orientation. Fingerprint identification is widely used in personal identification as it works well in most cases. However, it is difficult to acquire minutiae fingerprint features for some class of persons such as manual labourers, elderly people. As a result, other biometric characteristics are receiving more attention. Moreover, additional biometric features, such as palm prints, can be easily integrated with the existing authentication system to provide improved level of acuracy in personal authentication.

Corresponding Author: Mathivanan, B., Department of CSE, Sri Ramakrishna Engineering College, Coimbatore, India 


\subsection{Hand Geometry}

A hand geometry based recognition system works by capturing the image of a hand to determine the geometry and other metrics such as the length and width of fingers and other attributes. Some of the existing systems assess different parameters for effective and efficient recognition.

An important characteristic of geometry based approach is the postulation that an individual's hand does not drastically change after a certain age. Most of the existing systems use more number of attributes to describe a hand. Out of which, finger width may slightly vary over time. Including such attributes in the process of distance metric will notably reduce the accuracy of the system during practical implementation. So, in this study we consider only some of the selected attributes which will not change significantly over short periods of time.

\subsection{Palm Side of the Hand and Dorsum of Hand}

There are two sides in a hand. One is well known as palm print side or simply palm side of the hand. The other side or opposite side of the palm side of the hand is refereed in a very common way as "back side" of the hand or "top side" of the hand. In this study, we refer it as "Dorsum of Hand" since the word "Dorsum" is technically used in some literature to refer "back/top" of a body part.

Most of the earlier works (Zhang et al., 2003; Yoruk et al., 2006) including our previous works (Selvarajan et al., 2007; 2008a; 2008b; Oden et al., 2003) address the way of using palm side of the hand to extract different features of the hand and use those features to design biometrics based human recognition systems.

Even some of the earlier works (Lin et al., 2011) and the equipment they used for capturing the top side /dorsum of hand image, generally, capture the top view of the hand image and detect the different geometric features from it and use those features to design biometrics based human recognition systems.

\subsection{Advantages of Using Dorsum of Hand Image}

- Dorsum of hand is easy to capture using a simple device like an ordinary digital camera. And even consume lesser time than that of the scanning device which is generally used for acquiring palm side of the hand. So it will reduce the enrollment and verification time considerably

- During the acquisition of Palm Print side of the image using a scanner, the Hand pressure will distort the image considerably and even make some portions to look very flat-but this will not be in the case of using Dorsum of Hand image

- Since the dirt and sweat on the palm will spoil the clean surface of the scanner which is used for capturing the palm side of the hand. But in the case of, the device used for capturing dorsum of hand, the hand will not spoil the capturing device even at extremely dirty conditions. So the capturing device will not need frequent cleaning

- Even there is a possibility of adding the skin color of the hand as an additional attribute while using dorsum of hand

- During using the dorsum of hand, there is a possibility to find the absolute length of the finger by subtracting the length of finger nails. It is not possible while using the palm side of the hand image

- Dorsum of Hand will not be dirty than that of the palm side of the hand even in dirty work environment

- Since the blood most of the vessels and veins are very near at the surface of the dorsum of hand, there is a possibility of adding IR imaging technique to differentiate the real human hand from the fake imprints which will be used to attack the system. Even a sophisticated camera can be used to acquire both the normal as well as IR image

\subsection{Finger Knuckle Print (FKP)}

Recently, it is found that the FKP, which refers to the inbuilt patterns of the outer surface around the phalangeal joint of one's finger, is highly unique and can serve as a distinct biometric identifier. Abundant line-like textures are contained in an FKP image (Lin et al., 2010).

In a previous knuckle print based recognition system (Lin et al., 2009a; 2009b), the authors used a separate, special device for capturing the knuckle print of the persons.

But in this study, we propose a new hybrid model for biometrics based recognition system using Geometry of dorsum of hand and finger-knuckle-print. We are deriving different kind of geometric features of the hand as well as the knuckle print of the hand just by using a single snapshot of dorsum of hand image.

The knuckle print attained in this method will be arguably more constant and better than the previous method (Lin et al., 2011) and irrespective of the image capturing process. Because, in the previous method (Lin et al., 2010), the finger was kept in a bent position on the scanner, so that, the image will depend up on the different pressure exerted by the individual during bending the finger while orienting it on the scanner. This bending of finger will create some elongation in knuckle prints. But in this proposed method, the whole hand is kept in a flat and resting position. So there will not be any elongation in knuckle prints.

\subsection{The Design of the Proposed System}

The hand geometry images can be extracted from a hand image in a single top view image of the hand captured from the top mounted camera. Unlike other multi-biometrics systems a user need not to undergo through multiple sensors. Furthermore, the fraud 
associated with fake hand, can be reduced with the integration of knuckle print features. This study proposed a new method for person authentication using knuckle print and hand geometry features that are simultaneously acquired from a single hand image.

In this study, a biometric verification system based on hand geometry and knuckle print is designed. This system uses natural fusion approach as both biometric features originate from the same side of the hand image.

Unlike other multimodal biometric systems, only a single image capturing device is needed in this system to acquire input images. So, the users need not to go through the inconvenience of using several different acquiring devices for security access.

Steps involved in recognition.

\subsection{It Includes the Following Steps:}

- Image preparation

- Image preprocessing

- Segmentation

- Hand geometrical feature extraction

- Knuckle print extraction

- Matching process

\subsection{Image Preparation}

Initially the hand images are obtained from the selected user group and stored in the database. For that purpose, we used normal digital camera which is mounted in a special arrangement. The hand geometry samples were captured at 72 DPI resolution.

During the time of enrolling the users and preparing the dataset, the users are asked to place the hand stretched as much as possible on a single guide line drawn on the black surface on which the camera is mounted. The Guideline was used only to place the middle finger of the hand. The hand images of the same person were taken three times in different time intervals. We took it in three consecutive weeks.

We used the actual data set which we captured as the training data set. During evaluating the algorithm, we synthetically added random noise with the detected attribute set to mimic the difference in accuracy over time and tried to identify the hand of the noisy feature in features extracted from the original data set. The Fig. 1-3 shows one such example of the image of dorsum of hand.

\subsection{Image preprocessing}

In this stage, the input hand image is preprocessed with the objective of adjust the contrast and brightness of input image. Then, median filter is used to remove the "Salt and Pepper" type noises from the preprocessed image. It reduces the blurring of edges. Further, some unwanted portions of the original images were cropped automatically, resulting in clear hand images of uniform size. The images were then scaled down to a suitable size, smaller than the original size for handling them with better performance in terms of speed. The Fig. 2 shows one such cropped, Region Of Interest (ROI) hand image.

\subsection{Segmentation \\ 1.10. (A) Hand segmentation}

For the purpose of extracting the hand geometrical features, we have to do a binary segmentation of the image to distinguish the hand only area from its background. For this purpose, in our earlier work we addressed a simple and very fast algorithm for hand image segmentation using filtering, edge detection and region labeling techniques (Gonzalez and Woods, 2009; Milan et al., 1999). But that algorithm was not suitable for segmenting the image of dorsum of hand. The reasons are:

- The palm side of the image will be almost homogeneous. But the dorsum of hand image is not homogeneous due to the nature of different skin tones in different areas of the dorsum of hand.

- And most importantly, the area of the nails and the different knuckle print areas of hand will be distinguishably in different shade

- Further, the difference in the shades of skin colors among different people is very much deviating from the difference of shade of palm side of the same people

The above facts make the design of the segmentation algorithm little bit complex.

\subsection{Clustering Pixels Using RGB Values}

The following text explains the normal way of segmenting the colour image using the RGB coour space.

First, an colour hand image is taken as an input. The input image is in pixels form and is transformed into a RGB feature space.

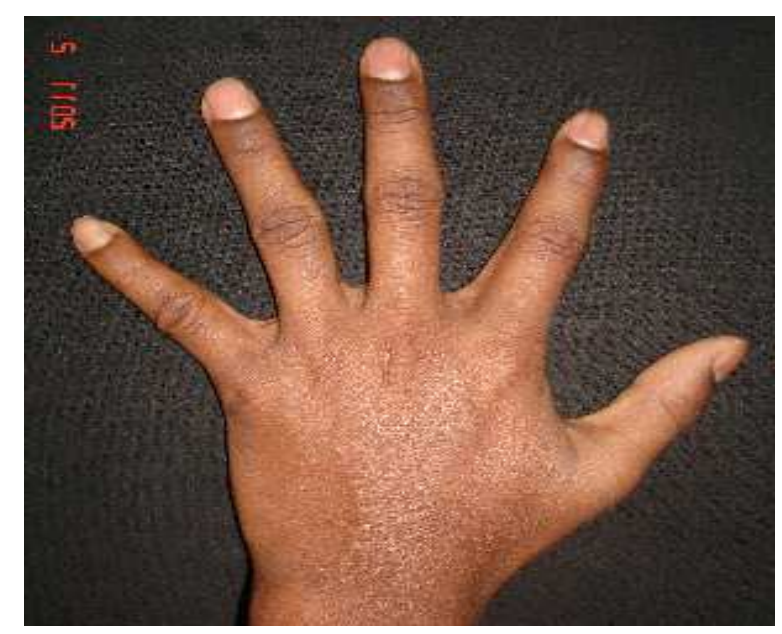

Fig. 1. The input hand image 


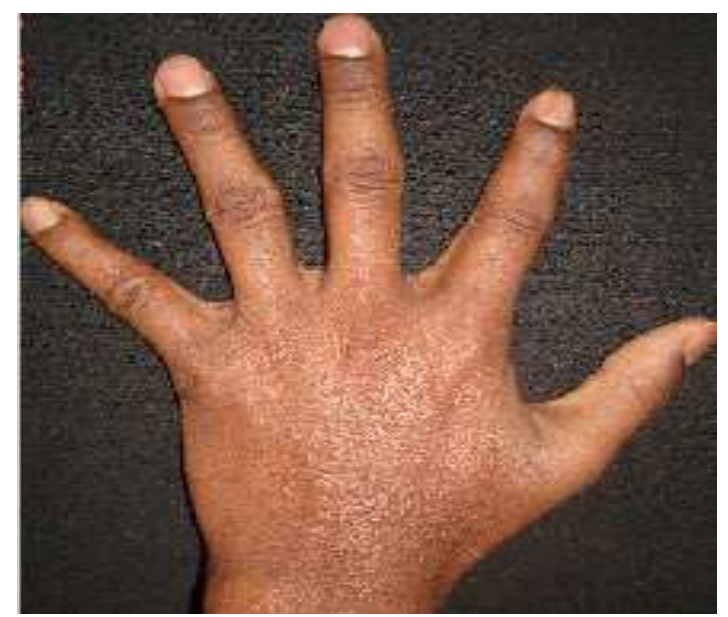

Fig. 2. The ROI of input hand image

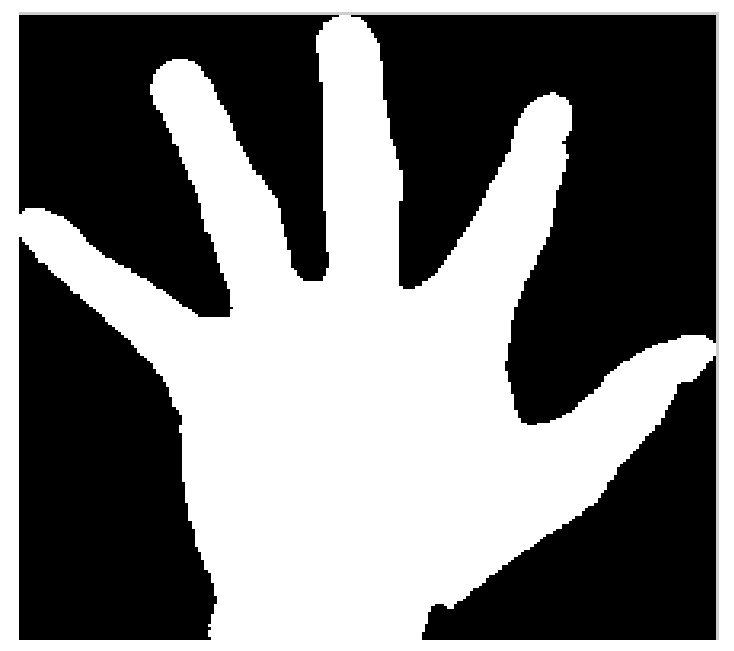

Fig. 3. The segmented hand image

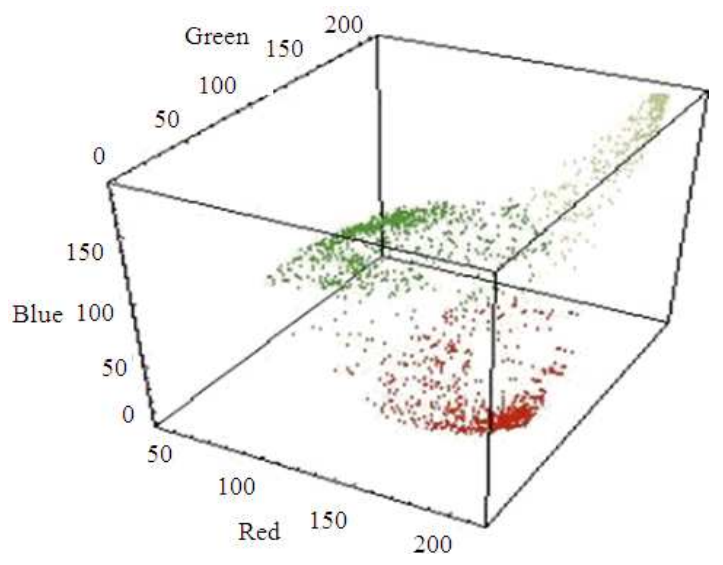

Fig. 4. The pixels in colour space
Next similar data points, i.e., the points which have approximately similar color, are grouped together using any clustering method. A clustering method such as kmeans clustering is used to form clusters as shown in the Fig. 4 the distances are calculated using Mahalanobis or Euclidean distant.

The Fig. 4 shows an example for how the data points are clustered in the 3-D RGB space. The data points with minimum Mahalanobis distance or Euclidean distance are grouped together to form the clusters.

Let us assume a colour image as a set of $n$ colour pixels, then we can represent it as follows:

$$
I=\left\{\left(r_{1}, g_{1}, b_{1}\right),\left(r_{2}, g_{2}, b_{2}\right), \ldots \ldots\left(r_{n}, g_{n}, b_{n}\right)\right\}
$$

So, using any clustering algorithms, we can cluster this set of $n$ pixel values. In this hand image segmentation, we have to segment the background and the hand image. So we have to segment the set of pixels in to two groups.

\subsection{The Proposed Segmentation Method}

We can represent the three layer of the color image (Red, Green and Blue layers) seperately as follows:

$$
\text { The image } \mathrm{I}=\{\mathrm{R}, \mathrm{G}, \mathrm{B}\}
$$

Each layer R, G and B can be treated as three separate gray image and we can apply image processing techniques on them.

The median filter is an effective method that can bring under control isolated noise without blurring sharp edges. Particularly, the median filter replaces a pixel by the median of all pixels in the neighborhood:

$$
y,[m, n]=\operatorname{median}\{x[I, j],(I, j) \in w\}
$$

where, $\omega$ represents a neighbourhood centered around location $(m, n)$ in the image.

We applied median filter separately on the three layers of the hand image and it will give the new image,

The median filtered Image $\mathrm{Im}=\{\mathrm{Rm}, \mathrm{Gm}, \mathrm{Bm}\}$

After this operation, each layer scaled differently by multiplied each values by a different factor.

Since there is 256 levels in each layer, we can calculate these multiplication factor as follows:

$$
\mathrm{f}_{\mathrm{r}}=1, \mathrm{f}_{\mathrm{g}}=256 / 2, \mathrm{f}_{\mathrm{b}}=256,
$$

If we multiply each layer values with these factors, then all the three layers will be scaled.

$$
\mathrm{Im}=\{\mathrm{Rm}, \mathrm{Gm}, \mathrm{Bm}\}
$$

So the resultant image layers scaled differently can be represented as follows: 


$$
\mathrm{I}_{\mathrm{S}}=\left\{\mathrm{Rm} * \mathrm{f}_{\mathrm{r}}, \mathrm{Gm} * \mathrm{f}_{\mathrm{g}}, \mathrm{Bm} * \mathrm{f}_{\mathrm{b}}\right\}
$$

Since the pixel values are scaled differently, the distance metric used in clustering algorithm leads to better segments.

The following Fig. 5 shows the difference in performance of the normally clustered pixels and the differently scaled pixels using k-mean clustering algorithm. The images used to show the difference in performance were somewhat poorly captured ones. Due to bad camera settings (or the texture of black cloth materiel used in the background), the lighting in the background is not uniform and it is very bad near the thumb of the hand image. The normal method was not able to identify the correct segments. But the proposed method was able to identify good segments.

\subsection{3. (B) Knuckle Print Segmentation}

To segment the knuckle Print image to separate the knuckle print lines and the background, simply, an efficient line/edge detection algorithm can be used. In this implementation, we used the Prewitt filter for this purpose. The Prewitt Edge filter is used to detect edges by applying a horizontal and vertical filter in sequence. Both filters are applied to the image and summed to form the final result.

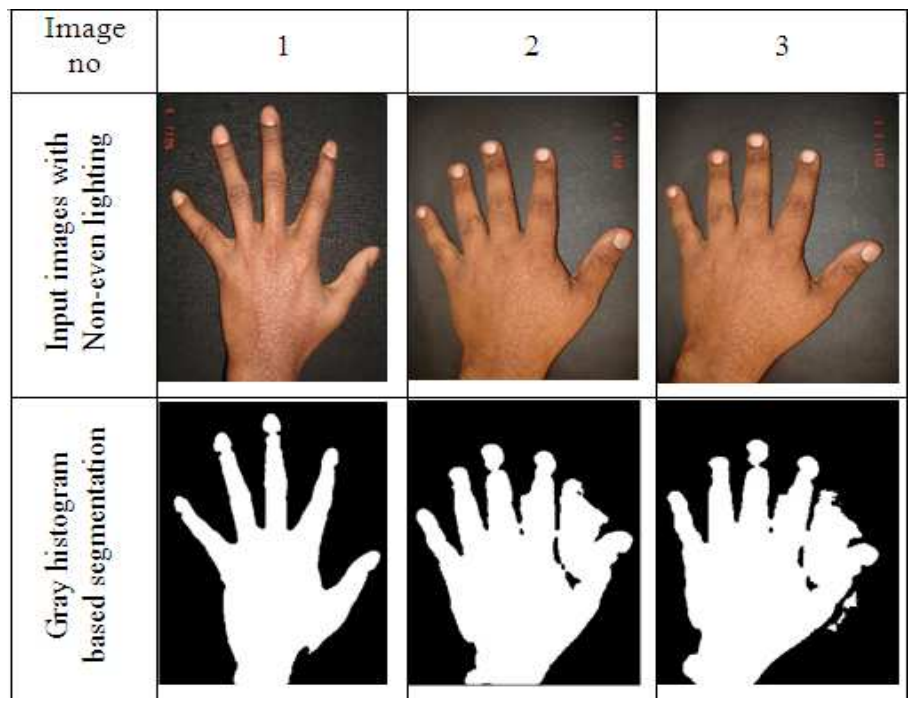

(a)

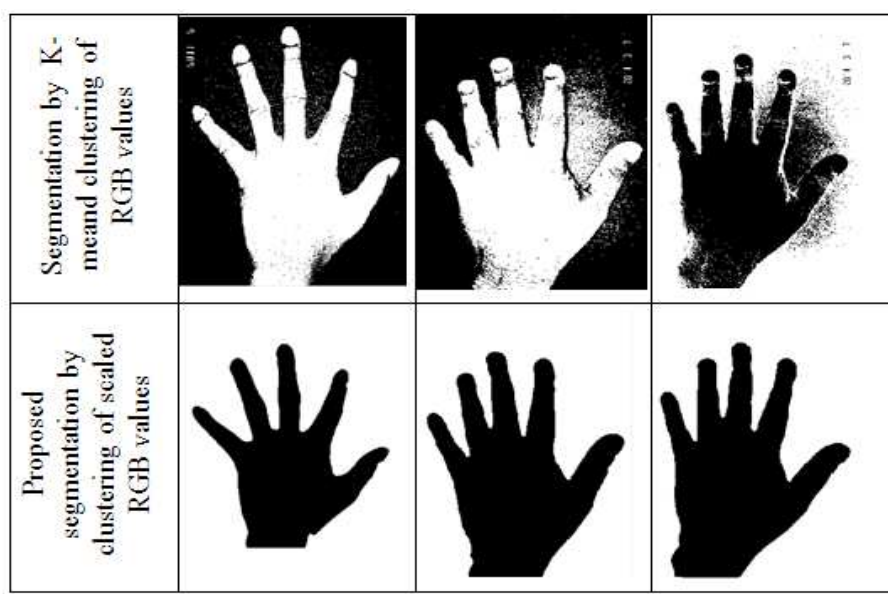

(b)

Fig. 5. Results of different Segmentation Methods 
Operator calculates the gradient of the image intensity at each point, giving the direction of the largest possible increase from light to dark and the rate of change in that direction. The result shows that how "abruptly" or "smoothly" the image changes at that point and therefore how likely it is that part of the image represents an edge, as well as how that edge is likely to be oriented. In practice, the magnitude (likelihood of an edge) calculation is more reliable and easier to interpret than the direction calculation.

Mathematically, the gradient of a two-variable function (here the image intensity function) is at each image point a $2 \mathrm{D}$ vector with the components given by the derivatives in the horizontal and vertical directions. At each image point, the gradient vector points in the direction of largest possible intensity increase and the length of the gradient vector corresponds to the rate of change in that direction. This implies that the result of the Prewitt operator at an image point which is in a region of constant image intensity is a zero vector and at a point on an edge is a vector which points across the edge, from darker to brighter values.

This filter uses two kernels during this operation. One $3 \times 3$ kernel is for changes in the image's horizontal direction and the another $3 \times 3$ kernels for changes in the image's vertical direction. The two $3 \times 3$ kernels are operated or convolved with the original knuckle print image to calculate the approximations of the derivatives.

If we define Gx and Gy as two images that contain the horizontal and vertical derivative approximations respectively, the computations are:

$$
\mathrm{G}_{\mathrm{x}}=\left(\begin{array}{ccc}
1 & 0 & -1 \\
1 & 0 & -1 \\
1 & 0 & -1
\end{array}\right) * A \text { and } \mathrm{G}_{\mathrm{y}}=\left(\begin{array}{ccc}
-1 & -1 & -1 \\
0 & 0 & 0 \\
1 & 1 & 1
\end{array}\right) * A
$$

where, $\mathrm{A}$ is the original source image.

The $\mathrm{x}$ coordinate is defined as increasing in the right-direction and the $\mathrm{y}$ coordinate is defined as increasing in the down-direction. To compute $\mathrm{Gx}$ and Gy, we move the appropriate kernel (window) over the input image, computing the value for one pixel and then shifting one pixel to the right. Once the end of the row is reached, we move down to the beginning of the next row up to the bottom of the image.

\subsection{Feature Extraction}

The feature extraction technique is originally explained in our previous hand geometry based recognition systems. In this study, we improved that method for extracting the features from the dorsum of hand image.

\subsection{Hand Geometrical Feature Extraction}

Instead of using palm print, in this study, we used knuckle print of the middle finger as a important additional signature/attribute of the hand. We extracted the knuckle print from immediately after the nail to the middle of the Dorsum of Palm. Because, knuckle prints could be found even on the middle portion of Dorsum of Palm (back side of palm/back side of hand). The Fig. 6 shows the extracted geometrical features from the hand image.

In addition to that, we addressed a method for finding finger length from the image and we propose the idea of including/excluding the finger nail length in the calculations of finger length.

In our previous hand geometry based recognition system, we used 14 attributes from the hand and the palm print of the hand image the 14 used attributes are:

- $\quad$ Finger tip lengths from a absolute reference point

- Valley point lengths from the absolute reference point

- 1 Left Middle reference point lengths from the absolute reference point

- Two Bottom reference point lengths from the absolute reference point

- Area of the hand image

- Circumference of the hand image

Finger lengths are measured from the absolute reference point, the length of valley point are also measured from the absolute reference point. The area of the hand image and circumference of the hand image are measured in pixels. Thus, the hand geometry of every hand image is characterized by a feature vector of lengths, circumferences and area.

In this proposed implementation, in addition to that 14 hand attributes we used some distinct set of attributed for better accuracy in recognition. They are:

- The lower width of the palm

- The middle width of the palm

- The circumference of the dorsum of palm

- The overall ROI circumference of the hand.

- The thumb joint width

- Small finger joint width

- 3 Valley to valley lengths

The following procedure will explain the geometry based hand attribute extraction as well as the Knuckle prints extraction method.

The binary image prepared from the segmented hand image was cropped exactly to contain the hand only area using the detected finger tips. The Fig. 2 shows the ROI of hand image. 


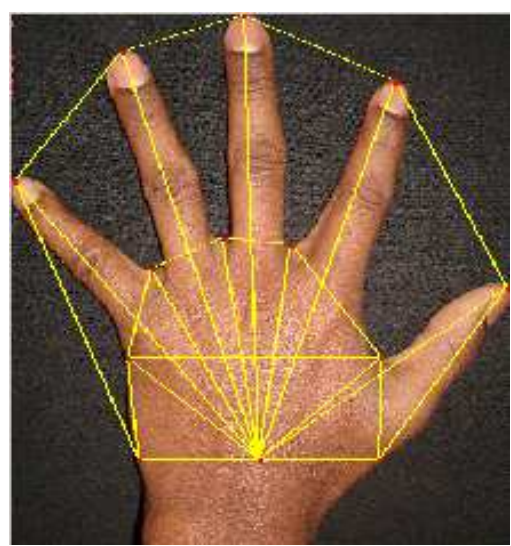

Fig. 6. The extracted hand attributes

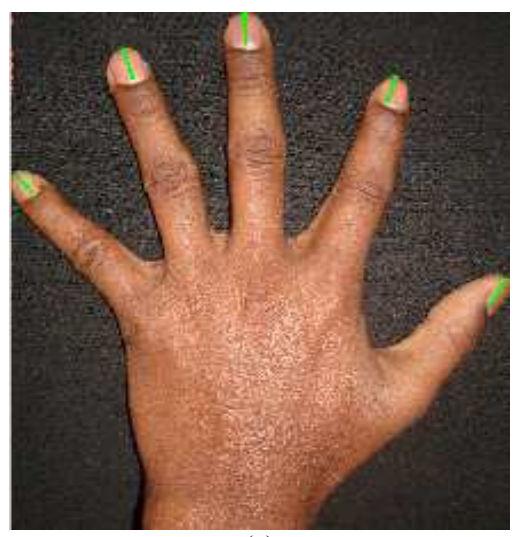

(a)

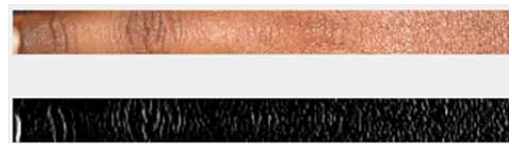

(b)

Fig. 7. The extracted middle knuckle print (a) the detected nail lengths (b) extracted knuckle print

After detecting the valley points, the main reference point above the wrist was detected using the valley point near the thumb finger as shown in Fig. 6. The main reference point can be considered as the absolute reference point since it is derived from the location of the valley point of the thumb finger and the width of the thumb finger. Further the reference point will not get affected even for any slightly different orientation of the thumb finger.

To detect the different points shown in the Fig. 6, the binary image was analyzed pixel by pixel several times, from top to bottom and left to right. The Fig. 6 shows a hand image with all the important detected attributes. In this Fig. 6, the yellow lines show the length of the different features.

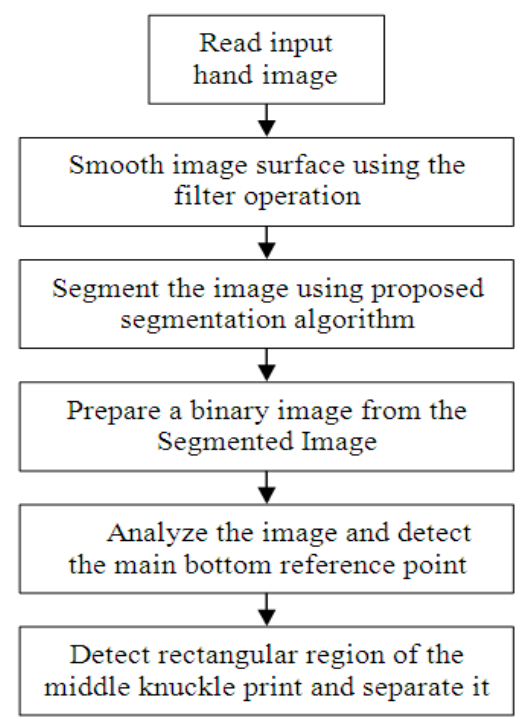

Fig. 8. Algorithm for knuckle print extraction

\subsection{Hand Knuckle Print Feature Extraction}

The Fig. 7 shows the automatically extracted knuckle print of the middle finger. The image has been roted to $90^{\circ}$ for the better visualization of the different bands or line features formed by the knuckle print. The algorithm for Knuckle Print extraction is shown in Fig. 8.

\section{MATERIALS AND METHODS}

\subsection{The Matching Policies}

The distance measure between the wrist points to finger tips is calculated using Euclidean distance.

Euclidean distance is most commonly used in clustering algorithm and calculating the distance between wrist point and finger tips. The Euclidean distance is the straight-line distance between two pixels.

\subsection{The Direct Euclidean Distance Based Model}

The following are the some of commonly used distance metrics in such matching policies.

$$
\text { Sum of difference: } \sum\left(D_{i}-T_{i}\right)
$$

Sum of Absolute Difference (SAD): $\sum_{i}\left|D_{i}-T_{i}\right|$

$$
\text { Sum of square of difference: } \sum_{i}\left(D_{i}-T_{i}\right)^{2}
$$

The weakness of this kind of distance metrics were discussed in several works. Even though they are weak in some aspect, they are very simple and direct methods in implementation point of view. 
Table 1. Recognition performance using Euclidean distance

\begin{tabular}{|c|c|c|c|c|c|}
\hline & \multirow[b]{2}{*}{$\begin{array}{l}\text { Total number of images } \\
\text { used in database }\end{array}$} & \multirow[b]{2}{*}{$\begin{array}{l}\text { Number of test } \\
\text { images used }\end{array}$} & \multicolumn{3}{|c|}{ Average $\%$ of positive recognition } \\
\hline & & & $\begin{array}{l}\text { Geometry based } \\
\text { attributes }\end{array}$ & $\begin{array}{l}\text { Knuckle print based } \\
\text { attributes }\end{array}$ & $\begin{array}{l}\text { Fusion of } \\
\text { both attributes }\end{array}$ \\
\hline 1 & 60 & 30 & 90 & 95.00 & 97.0 \\
\hline 2 & 80 & 40 & 90 & 95.00 & 97.0 \\
\hline 3 & 100 & 50 & 90 & 95.00 & 96.0 \\
\hline \multirow[t]{2}{*}{4} & 120 & 60 & 90 & 94.00 & 96.0 \\
\hline & Average & & 90 & 94.75 & 96.5 \\
\hline
\end{tabular}

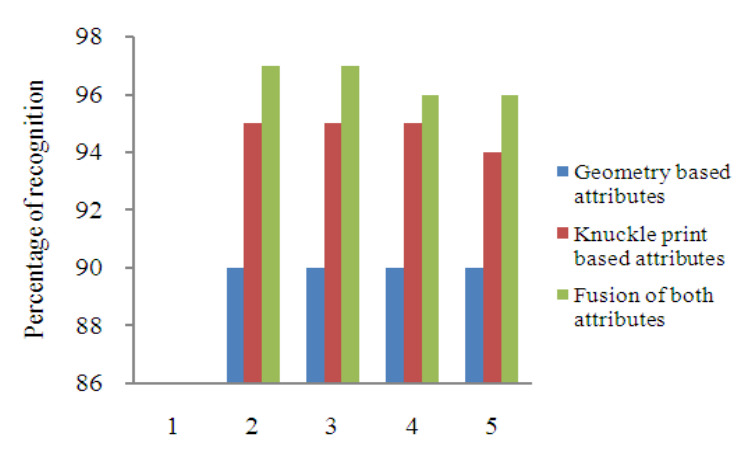

Fig. 9. Performance of recognition

Moreover, they also give better results. So in this study we will implement a simple Euclidean based distance metric based matching policy for comparison.

Euclidean distance: $=\sqrt{\left((\mathrm{x} 1-\mathrm{x} 2)^{2}+(\mathrm{y} 1-\mathrm{y} 2)^{2}\right)}$

where, $(x 1, y 1)$ and $(x 2, y 2)$ are two pixel points or two data points.

In most cases when people mention about distance, they refer to Euclidean distance. Euclidean distance or simply 'distance' examines the root of square differences between coordinates of a pair of objects.

\section{RESULTS AND DISCUSSION}

We have tested the algorithms and the hand recognition system on a normal desk top computer. The following Table 1 shows the performance of the different approaches. To evaluate the performance, noise was synthetically added with the detected geometry based training attributes (Fig. 9).

\section{CONCLUSION}

The objective of this study is to investigate the integration of geometrical features of dorsum of hand image along with the knuckle print features and to achieve higher performance that may not be possible with single biometric indicator alone as well as the previous hand based recognition methods.

The experimental results also suggest the usefulness of several geometrical features of the hand which can be effectively used in hand-shape based human recognition.

\section{REFERENCES}

Gonzalez, R.C. and R.E. Woods, 2009. Digital Image Processing. 3rd Edn., Pearson Education India, ISBN-10: 8131726959, pp: 954.

Lin, Z., L. Zhang and D. Zhang, 2009a. Finger-knuckleprint: A new biometric identifier. Proceedings of the 16th IEEE International Conference on Image Processing, Nov. 7-10, IEEE Xplore Press, Cairo, pp: 1981-1984. DOI: 10.1109/ICIP.2009.5413734

Lin, Z., L. Zhang and D. Zhang, 2009b. Finger-knuckleprint verification based on band-limited phase-only correlation. Comput. Anal. Images Patt., 5702: 141148. DOI: $10.1007 / 978-3-642-03767-2-17$

Lin, Z., L. Zhang, D. Zhang and H. Zhu, 2010. Online finger-knuckle-print verification for personal authentication. Patt. Recogn., 43: 2560-2571. DOI: 10.1016/j.patcog.2010.01.020

Lin, Z., L. Zhang, D. Zhang and H. Zhu, 2011. Ensemble of local and global information for finger-knuckleprint recognition. Patt. Recogn., 44: 1990-1998. DOI: $10.1016 /$ j.patcog.2010.06.007

Milan, S., V. Hlavac and R. Boyle, 1999. Image Processing Analysis and Machine Vision. 2nd Edn., PWS Pub., Pacific Grove, ISBN-10: 053495393X, pp: 770.

Oden, C., A. Ercil and B. Buke, 2003. Combining implicit polynomials and geometric features for hand recognition. Patt. Recogn. Lett., 24: 21452152. DOI: $10.1016 / \mathrm{S} 0167-8655(03) 00087-4$

Selvarajan, S., V. Palanisamy and B. Mathivanan, 2007. Segmentation of hand images for hand geometry based human identification and recognition. GESTS Int. Trans. Comput. Sci. Eng., 43: 152-162.

Selvarajan, S., V. Palanisamy and B. Mathivanan, 2008a. Hand geometry based human identification and recognition system using some selected hand attributes. Int. J. Syst. Cybernetics Inform.

Selvarajan, S., V. Palanisamy and B. Mathivanan, 2008b. Hand geometry based human identification and recognition system using more significant hand attributes. Int. Eng. Technol. J. Adv. Comput., 2: 69-73.

Yoruk, E., E. Konukoglu, B. Sankur and J. Darbon, 2006. Shape-based hand recognition. IEEE Trans. Image, 15: 1803-1815. DOI: 10.1109/TIP.2006.873439

Zhang, D., W.K. Kong, J. You and M. Wong, 2003. Biometrics-online palmprint identification. IEEE Trans. Patt. Anal. Mach. Intell., 25: 1041-1050. 